\title{
Efecto antinociceptivo y antiinflamatorio de la metformina en modelos experimentales en ratón
}

Sánchez-Gavidia Joseph 1,2,3,5, Pante-Medina Carlos ${ }^{1}$, Lujan-Carpio Elmer ${ }^{1,4}$, Salazar-Granara Alberto* 1,3,5

RESUMEN

Objetivo: Analizar la actividad antiinflamatoria y antinociceptiva de la metformina en un evento agudo de inflamaciónedema y dolor inducido en roedor.

Materiales y métodos: Se utilizaron 198 ratones hembras. Se evaluó el efecto antiinflamatorio con la prueba de edema plantar inducido por carragenina en 64 ratones y, para evaluar el efecto sobre el dolor, se utilizó la prueba de placa caliente e inmersión de la cola, con 64 y 70 ratones, respectivamente. En ambos casos se tuvieron grupos controles, placebo y experimentales. Para el análisis se emplearon las pruebas de Shapiro-Wilk, de ANOVA de una cola, de Tukey y correlación de Pearson.

Resultados: En el edema de la pata por carragenina, los grupos metformina 50 y $150 \mathrm{mg} / \mathrm{kg}$ presentaron efecto antiinflamatorio a la segunda y tercera hora. En la prueba de placa caliente, los grupos metformina 100,200 y $250 \mathrm{mg} / \mathrm{kg}$ presentaron efecto analgésico en la segunda y cuarta hora. En la prueba de inmersión de cola, los grupos metformina 100, 150,200 y $250 \mathrm{mg} / \mathrm{kg}$ presentaron efecto analgésico desde el minuto 15 hasta terminar el experimento; además, el grupo metformina $150 \mathrm{mg} / \mathrm{kg}$ tuvo efecto estadísticamente equivalente al grupo control de morfina $10 \mathrm{mg} / \mathrm{kg}$.

Conclusiones: Se demuestra la actividad antiinflamatoria y antinociceptiva aguda de metformina a diferentes dosis, en modelos experimentales con ratones.

Palabras clave: Metformina; Inflamación; Dolor; Carragenina; Sistema nervioso central (Fuente: DeCS BIREME).

\section{Antinociceptive and anti-inflammatory effect of metformin in experimental models conducted in mice}

ABSTRACT

Objective: To analyze the anti-inflammatory and antinociceptive activity of metformin in an acute event of inflammation-edema and pain induction in rodents.

Materials and methods: One hundred ninety-eight (198) female mice were used. The anti-inflammatory effect was evaluated in 64 mice using the carrageenan-induced paw edema test, and the antinociceptive effect was assessed in 64 and 70 mice using the hot plate test and the tail immersion test, respectively. In both cases, there were control, placebo and experimental groups. For the analysis, the Shapiro-Wilk test, one-way ANOVA test, Tukey test and Pearsonian coefficient of correlation were used.

Results: In the carrageenan-induced paw edema test, the metformin 50 and $150 \mathrm{mg} / \mathrm{kg}$ groups showed an antiinflammatory effect at the second and third hours. In the hot plate test, the metformin 100, 200 and $250 \mathrm{mg} / \mathrm{kg}$ groups showed an antinociceptive effect at the second and fourth hours. In the tail immersion test, the metformin $100,150,200$ and $250 \mathrm{mg} / \mathrm{kg}$ groups showed an antinociceptive effect from minute 15 until the end of the experiment. In addition, the metformin $150 \mathrm{mg} / \mathrm{kg}$ group had a statistically equivalent effect to the morphine $10 \mathrm{mg} / \mathrm{kg}$ control group.

Conclusions: The acute anti-inflammatory and antinociceptive activity of metformin at different doses is demonstrated in experimental models conducted in mice.

Keywords: Metformin; Inflammation; Pain; Carrageenan; Central nervous system (Source: MeSH NLM).

1. Universidad de San Martín de Porres, Facultad de Medicina Humana, Centro de Investigación de Medicina Tradicional y Farmacología. Lima, Perú.

2. Universidad de San Martín de Porres, Facultad de Medicina Humana, Sección de Posgrado, Programa de Maestría en Ciencias Básicas Médicas. Lima, Perú.

3. Sociedad Peruana de Farmacología y Terapéutica Experimental SOPFARTEX. Lima, Perú.

4. Sociedad Científica de Estudiantes de Medicina de la Universidad de San Martín de Porres. Lima, Perú.

5. Asociación Médicos de Vida del Perú. Lima, Perú.

*Autor corresponsal 


\section{INTRODUCCIÓN}

El proceso de desarrollo de un fármaco toma un largo tiempo, acarrea altísimo costo y, además, de miles de moléculas, solo una es aprobada por la autoridad competente para su posterior comercialización. En el mercado, el uso y el tiempo revelan la efectividad o no de la droga, con consecuencias económicas a favor o en desmedro de la industria farmacéutica ${ }^{(1,2)}$.

De acuerdo a lo descrito, es importante optimizar el uso de las drogas que actualmente se emplean, y una posibilidad es buscar aplicaciones fuera de indicación (off-label), esto se basa en la probabilidad de efectos pleiotrópicos, lo cual significa que el medicamento es capaz de presentar otros efectos farmacológicos, diferentes al de su mecanismo principal ${ }^{(3-5)}$.

Diversos medicamentos, a través del tiempo, están revelándose como drogas pleiotrópicas. Un ejemplo es el ácido acetil salicílico (aspirina), que surgió como un antiinflamatorio, y, al que hoy en día, se le atribuyen efecto antipirético, analgésico, antiplaquetario, anticancerígeno, entre otros ${ }^{(4,6)}$.

Por otra parte, revelar efectos pleiotrópicos en una droga, redundaría en reducir costos a favor del paciente, particularmente, en aquellos que usaran crónicamente el medicamento, como los pacientes con diabetes mellitus, hipertensión arterial, enfermedades mentales, infección por VIH-SIDA, etc.

Particularmente, una potencial droga con efectos pleiotrópicos es la antidiabética metformina, uno de los medicamentos más recetados para el manejo de la diabetes mellitus en el mundo ${ }^{(7)}$.

Investigaciones clínicas han demostrado que el uso de metformina, además de disminuir los niveles de glicemia y hemoglobina glicosilada ${ }^{(8)}$, reduce las complicaciones cardiovasculares de la diabetes y su mortalidad asociada (9). En el tratamiento de ovario poliquístico, al asociarse con cambios de estilo de vida y anticonceptivos orales, presenta disminución de grasa de distribución central, reducción total de testosterona y aumento de $\mathrm{HDL}^{(10)}$, mejora la función endotelial (11) y tiene acción antiinflamatoria al disminuir diferentes mediadores como son las moléculas de adhesión, citocinas (TNF, IL-6, IL-1), factores de migración de macrófagos, proteína $C$ reactiva, entre otros ${ }^{(12)}$. Algunos datos preclínicos señalan una acción anticancerígena ${ }^{(13)}$.

El dolor presenta un componente de nocicepción, que se inicia a partir de la activación de un nociceptor y, a partir de este, se desencadena la vía del dolor. Asimismo, la inflamación presenta tres componentes clásicos (dolor, calor y edema). Existen modelos experimentales en roedores que permiten diferenciar la actividad aguda antinociceptiva, a partir de la exploración de la vía del dolor y también, discriminar la acción antinflamatoria, a partir de la exploración del edema ${ }^{(14)}$.

Un estudio exploró la actividad analgésica y antiinflamatoria de la metformina en un modelo experimental en roedores mediante el test de formalina que tiene tres etapas ${ }^{(15)}$. En la primera, se explora la potencial actividad antinociceptiva, sin embargo, la presencia de mediadores de la inflamación limita la conclusión a esta acción farmacológica. La segunda fase evalúa la inhibición de la inflamación, en relación a la intensidad del dolor, pero no explora la magnitud de la antiinflamación en el componente del edema, que acompaña la triada con dolor y calor.

Con base en los antecedentes, el presente estudio tiene como propósito determinar la actividad antiinflamatoria de la metformina en roedores. Para el componente del edema se empleará el test de inducción de edema por carragenina; y para la actividad antinociceptiva, los test de inmersión de la cola y de la placa caliente.

\section{MATERIALES Y MÉTODOS}

Diseño y población de estudio

Estudio cuasi-experimental y doble ciego, que se realizó en el Centro de Investigación de Medicina Tradicional y Farmacología, de la Facultad de Medicina Humana, de la Universidad de San Martin de Porres (FMH-USMP).

La población animal se conformó por 198 ratones albinos hembras Mus musculus, adquiridos en el Centro Nacional de Productos Biológicos del Instituto Nacional de Salud, (INS - Bioterio, Chorrillos, Lima-Perú), con pesos entre 25 y 35 gramos. Los ratones pasaron por una etapa de aclimatación en las instalaciones de la FMH-USMP. Fueron alojados 4 roedores por jaula, y se mantuvieron las siguientes condiciones: temperatura de $22{ }^{\circ} \mathrm{C}(+/-3)$, humedad entre $45-70 \%$, ciclos de luz/oscuridad de 12 horas y niveles de sonido menores de $70 \mathrm{~dB}$. Asimismo, los ratones recibieron agua ad libitum y alimentación balanceada, y fueron privados de alimentos 12 horas antes del experimento.

Para la muestra química se utilizó metformina en tableta de $500 \mathrm{mg}$ (lote 1010135, EXP.: 01/2016), ampolla de diclofenaco $75 \mathrm{mg} / 3 \mathrm{ml}$ (código A021143, RS: EG-2537), morfina clorhidrato solución inyectable (- R.S. No Ng-1393Laboratorio Unidos S. A. Lote 008459, EXP.: 08/2016), carragenina lambda $25 \mathrm{~g}$ (code $80 \mathrm{~K}$ 1334). 
Variables y mediciones

Prueba de actividad antiinflamatoria por edema plantar inducido por carragenina en ratones (carragenan paw oedema test): Consiste en la administración subcutánea de $0,5 \mathrm{ml}$ de una solución de carragenina lambda al $3 \%$, a nivel de la aponeurosis plantar del ratón. La pata de los ratones es sumergida en el pletismometro digital que contiene agua exactamente hasta la marca de tinta en la piel sobre el maléolo lateral y se registran los volúmenes obtenidos. En este caso, se registró el volumen cada hora durante 5 horas. El porcentaje de inhibición de edema es controlado para cada grupo de animales en comparación con el grupo control. Para el cálculo del porcentaje de inhibición se utilizó la siguiente fórmula: $((\mathrm{Vc}-\mathrm{Vt}) / \mathrm{Vc}) \mathrm{x}$ 100 , donde Vc es la diferencia de volumen control y Vt es la diferencia de volumen tratado en el mismo tiempo.

Prueba de la placa caliente en ratones (hot-plate test): En esta prueba se introduce a un ratón en un cilindro abierto colocado sobre un suelo metálico calentado a una temperatura constante. Se mide el tiempo de latencia hasta la expresión de dos respuestas conductuales: el lamido de las patas traseras y el salto. En ocasiones se toma en cuenta solo la primera que se produzca. Es una prueba muy sensible al aprendizaje, de tal forma que no es recomendable su repetición ${ }^{(14)}$. En este estudio se registró el parámetro de tiempo de reacción (TR) (en segundos) frente al estímulo térmico. Primero se tomó la medición del basal correspondiente para todos los grupos experimentales sin tratamiento. Luego se realizó la medición y registro de los tiempos de reacción de los ratones frente al estímulo térmico a partir de la primera hora hasta la cuarta. Dicha medición y registro se realizaron sin conocer a qué grupo correspondía cada ratón evaluado.

Prueba de inmersión de cola en ratones (tail-immersion test): Consiste en la inmersión de la cola del ratón en un recipiente con agua a $55^{\circ} \mathrm{C}$. Se deja al ratón en una jaula antes de la prueba por 30 minutos para que se adapte. Se marca los $5 \mathrm{~cm}$ distales de la cola como punto de referencia y esta es inmersa en un recipiente con agua a $55{ }^{\circ} \mathrm{C}$, hasta que el ratón reacciona en los próximos segundos retirando la cola. Se realiza la evaluación antes y después de administrar por vía oral el compuesto de referencia o controles positivos (a los $0,15,30 \mathrm{~s}, 45,60$ y 120 minutos). El tiempo máximo de inmersión es de 5 segundos en ratones que no han recibido algún compuesto y 15 segundos en los que sí recibieron.

Formación de grupos experimentales

En la prueba de edema inducido por carragenina y la prueba de placa caliente, se formaron 8 grupos de 8 ratones cada uno: diclofenaco $(8 \mathrm{mg} / \mathrm{kg})$, agua destilada $(1 \mathrm{ml} / 10 \mathrm{~g}$ de peso corporal), blanco (que no recibió ninguna sustancia), y 5 grupos con metformina a dosis de 50, 100, 150, 200 y $250 \mathrm{mg} / \mathrm{kg}$. En la prueba de inmersión de la cola, los animales se dividieron en 7 grupos de 10 ratones cada uno: morfina $(10 \mathrm{mg} / \mathrm{kg})$, agua destilada $(1 \mathrm{ml} / 10 \mathrm{~g}$ de peso corporal), y 5 grupos con metformina a dosis de $50,100,150,200$ y $250 \mathrm{mg} / \mathrm{kg}$, respectivamente. Para la distribución de los roedores en cada grupo se realizó una prueba simple randomizada por método de sorteo.

Se aplicó un sistema de doble ciego en que el encargado de administrar las soluciones y el que observó las reacciones no conocía el origen de las soluciones (16). La humedad fue monitorizada con el higrómetro digital modelo VWR Thomas Traceable ${ }^{\circledR}$, con capacidad de medir la humedad de $60 \%$ a $82 \%$. La temperatura de la habitación se controló con el medidor de temperatura 35519-045 con rango de medición de $5{ }^{\circ} \mathrm{C}$ a $34{ }^{\circ} \mathrm{C}$. Para el sonido, se utilizó el sensor de sonido digital Radio Shack 33-2055 con capacidad de medir de 60 a $120 \mathrm{~dB}$. La temperatura de la habitación se mantuvo estable con 2 calentadores marca NF15C 1500 WImaco con 2 niveles de intensidad (1000-2000 W) para un área de $15 \mathrm{~m}^{2}$. Para las pruebas se utilizó el pletismómetro LETICA digital (LE-750) y la placa caliente Thermo Scientific ${ }^{\mathrm{TM}} \mathrm{Cimarec}^{\mathrm{TM}}\left(5-540{ }^{\circ} \mathrm{C}\right)$. Se entrenó a los investigadores para identificar las manifestaciones físicas con el software Virtual Pharmacology Lab ${ }^{(17)}$, Microlabs ${ }^{(18)}$, $\mathrm{y}$ un piloto in vivo.

\section{Análisis estadístico}

Se aplicaron las pruebas de normalidad de Shapiro-Wilk, de ANOVA de una cola, de Tukey y la prueba de correlación de Pearson. Se consideró significancia estadística de $p<0.05$ con intervalo de confianza de $95 \%$. Como soporte informático se usó el programa estadístico Microsoft Office Excel 2013 y GraphPad Prism 5.01.

\section{Consideraciones éticas}

El estudio fue aprobado por el Instituto de Investigación de la FMH-USMP según los lineamientos del International Guiding Principles for Biomedical Research Involving Animal (19) y Ethical guidelines for investigations of experimental pain in conscious animals ${ }^{(20)}$.

\section{RESULTADOS}

Resultados para la prueba del edema plantar inducido por carragenina

En la tabla 1 se observa inhibición de la inflamación estadísticamente significativa por acción de la metformina (ANOVA de una cola $p<0,05$ ), que alcanzó el máximo nivel a las 2 horas en dosis de $50 \mathrm{mg} / \mathrm{kg}$, a las 3 horas en dosis de $100 \mathrm{mg} / \mathrm{kg}$, a las 4 horas en dosis de $150 \mathrm{mg} / \mathrm{kg}$, a las 3 horas a dosis de $200 \mathrm{mg} / \mathrm{kg}$, y a las 2 horas a dosis de 250 $\mathrm{mg} / \mathrm{kg}$. La prueba estadística de pareo post hoc de Tukey refrenda la inhibición de la inflamación a dosis de $50 \mathrm{mg} / \mathrm{kg}$ de metformina a la segunda y tercera $(p<0,05$ y $p<0,0$ 
respectivamente), y a dosis $150 \mathrm{mg} / \mathrm{kg}$ de metformina en la segunda hora $(p>0.05)$. Acorde a la relación dosis respuesta, no se encontró correlación, lo cual se corroboro mediante la prueba estadística de correlación de Pearson $(p>0,05)$.
Asimismo, se observó que la eficacia antinflamatoria fue superior para diclofenaco en la hora 2; sin embargo, en la hora 3 , metformina $50 \mathrm{mg} / \mathrm{kg}$ y $100 \mathrm{mg} / \mathrm{kg}$ superaron la eficacia antinflamatoria del diclofenaco (Figura 1).

Tabla 1. Porcentajes de inhibición del edema plantar por cada hora según la dosis

\begin{tabular}{|c|c|c|c|c|c|c|c|c|c|c|c|c|}
\hline \multirow{2}{*}{ Hora } & \multicolumn{2}{|c|}{$\begin{array}{c}\text { Metformina } \\
50 \mathrm{mg} / \mathrm{kg}^{*}\end{array}$} & \multicolumn{2}{|c|}{$\begin{array}{l}\text { Metformina } \\
100 \mathrm{mg} / \mathrm{kg}^{*}\end{array}$} & \multicolumn{2}{|c|}{$\begin{array}{l}\text { Metformina } \\
150 \mathrm{mg} / \mathrm{kg}^{*}\end{array}$} & \multicolumn{2}{|c|}{$\begin{array}{l}\text { Metformina } \\
200 \mathrm{mg} / \mathrm{kg}^{*}\end{array}$} & \multicolumn{2}{|c|}{$\begin{array}{l}\text { Metformina } \\
250 \mathrm{mg} / \mathrm{kg}^{*}\end{array}$} & \multicolumn{2}{|c|}{$\begin{array}{c}\text { Diclofenaco } \\
8 \mathrm{mg} / \mathrm{kg}^{*}\end{array}$} \\
\hline & $\%$ iN & $\% \mathrm{iP}$ & $\%$ iN & $\%$ iP & $\%$ iN & $\%$ iP & $\%$ iN & $\%$ iP & $\%$ iN & $\%$ iN & $\%$ iN & $\%$ iN \\
\hline 1 & 21,8 & 30,6 & 19,7 & 28,7 & 36,6 & 43,7 & 14,78 & 24,4 & 19 & 28,1 & 18,3 & 27,5 \\
\hline 2 & $53,9^{* *}$ & 48 & 9 & $-2,4$ & $41^{*}$ & 33 & 27,7 & 18,4 & 36,2 & 28 & 64 & 41 \\
\hline 3 & $46^{* * *}$ & 44,8 & 42,9 & 34 & 21,3 & 20,6 & 33,1 & 22,7 & 27,6 & 16,3 & $42,3^{*}$ & 33,3 \\
\hline 4 & 29,3 & 33,6 & 10 & 24,3 & 42,4 & 46,7 & -7 & 0,9 & $-27,3$ & $-17,7$ & 17,2 & 23,4 \\
\hline 5 & 20,5 & 22,4 & 24,5 & 26,4 & 19,7 & 21,6 & 5,9 & 9,6 & $-2,5$ & 0 & 10,6 & 28,8 \\
\hline
\end{tabular}

\% iN: \% inhibición respecto al grupo blanco, \% iP: \% inhibición respecto al grupo placebo (aguda destilada). *Prueba de ANOVA de una cola $p<0.05$. ${ }^{* *}$ Prueba de Tukey $p<0.05$. ${ }^{* * *}$ Prueba de Tukey $p<0.01$.

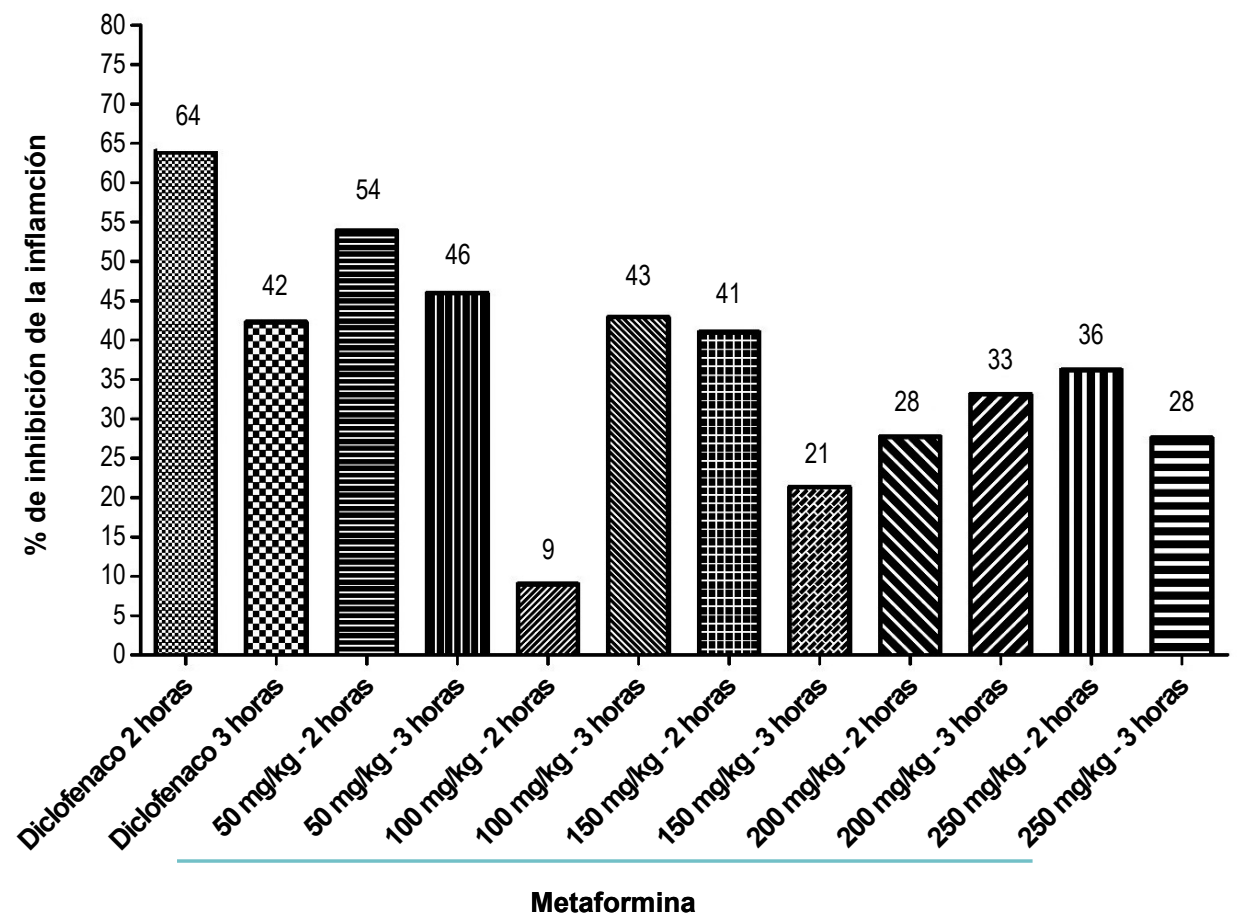

Tratamientos

Figura 1. Porcentajes máximos de inhibición del edema en la hora 2 y 3 según las dosis administradas 
Resultados para la prueba de la placa caliente (Hot Plate Test)

La prueba de ANOVA de una cola reveló significancia estadística $(p<0.05)$ para todos los grupos que recibieron metformina (Tabla 2); esto se interpreta como actividad antinociceptiva para los casos en que el tiempo de reacción o umbral de nocicepción se incrementa frente al basal o los grupos controles (blanco y placebo). La prueba estadística de pareo post hoc de Tukey refrenda la acción antinociceptíva de metformina $100 \mathrm{mg} / \mathrm{kg}$ en la segunda y cuarta hora, metformina $200 \mathrm{mg} / \mathrm{kg}$ y metformina $250 \mathrm{mg} /$ $\mathrm{kg}$ en la segunda hora, y diclofenaco $8 \mathrm{mg} / \mathrm{kg}$ en la cuarta hora $(p<0,05)$. En relación a la actividad dosis respuesta se observó una tendencia positiva, sin embargo, la prueba de correlación de Pearson reveló un valor p>0.05 (Figura 2).

Tabla 2. Tiempos de reacción en la prueba de la placa caliente por cada hora según la dosis

\begin{tabular}{|c|c|c|c|c|c|c|c|c|}
\hline Hora & $\begin{array}{c}\text { Metformina } \\
50 \text { mg/kg* } \\
\text { TR }\end{array}$ & $\begin{array}{l}\text { Metformina } \\
100 \mathrm{mg} / \mathrm{kg}^{*}\end{array}$ & $\begin{array}{l}\text { Metformina } \\
150 \mathrm{mg} / \mathrm{kg}^{*}\end{array}$ & $\begin{array}{l}\text { Metformina } \\
200 \mathrm{mg} / \mathrm{kg}^{*}\end{array}$ & $\begin{array}{l}\text { Metformina } \\
250 \mathrm{mg} / \mathrm{kg}^{*}\end{array}$ & $\begin{array}{c}\text { Diclofenaco } \\
8 \mathrm{mg} / \mathrm{kg}^{*}\end{array}$ & Blanco & Placebo \\
\hline $1 \mathrm{~h}$ & 13,4 & 17 & 13,8 & 14,8 & 21,2 & 14 & 17,5 & 12,9 \\
\hline $2 \mathrm{~h}$ & 12,4 & $13^{* *}$ & 18,4 & $21,6^{\star *}$ & $26,5^{\star *}$ & 19,5 & 11,4 & 14,6 \\
\hline $3 \mathrm{~h}$ & 18,8 & 16,3 & 17 & 19,1 & 18,8 & 24,3 & 17 & 19,4 \\
\hline $4 \mathrm{~h}$ & 14,4 & $17^{* *}$ & 17,6 & 23,6 & 24,3 & $20,1^{\text {** }}$ & 11,6 & 10 \\
\hline
\end{tabular}

TR: Tiempo de reacción en segundos al estímulo físico nociceptivo. *Prueba de ANOVA de una cola $p<0.05 .{ }^{* *}$ Prueba de Tukey $p<0.05$.

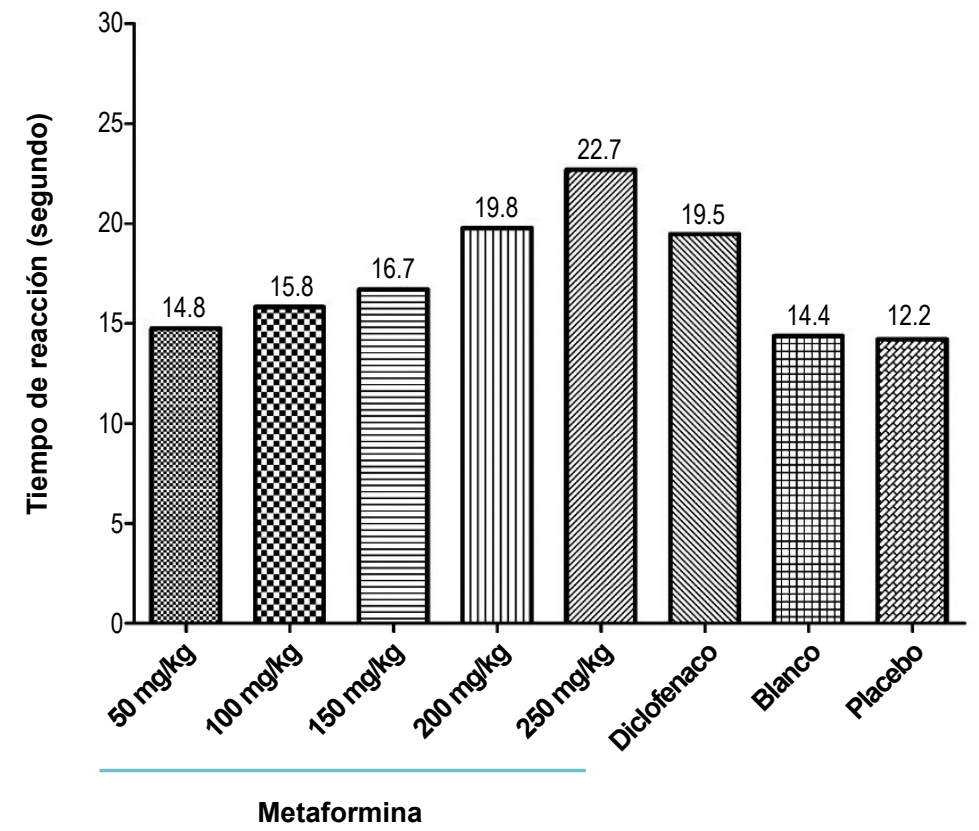

\section{Tratamientos}

Figura 2. Promedios de tiempo de reacción y dosis en la prueba de la placa caliente 
Resultados de la prueba de inmersión de cola (tail immersion test)

El tiempo de reacción (TR) equivale al umbral de nocicepción, tiempo en que el roedor retira la cola al estímulo físico térmico. En ese aspecto, la metformina, en todas sus dosis probadas, obtuvo un incremento del umbral de nocicepción, lo cual, según la prueba de ANOVA de una cola, fue estadísticamente significativo $(p<0.05)$, y se interpreta como actividad antinociceptíva (Tabla 3). No hay correlación dosis-efecto
( $p>0.05)$. La prueba estadística de pareo post hoc de Tukey, frente al placebo, refrendó la actividad antinociceptiva de metformina a dosis de $100 \mathrm{mg} / \mathrm{kg}, 150 \mathrm{mg} / \mathrm{kg}, 200 \mathrm{mg} / \mathrm{kg}$ y $250 \mathrm{mg} / \mathrm{kg}(\mathrm{p}<0.05)$. La prueba estadística de pareo post hoc de Tukey, frente a morfina, reveló actividad antinociceptíva equivalente de metformina en dosis de $150 \mathrm{mg} / \mathrm{kg}(p>0.05)$ (ver figura 3). En relación a la dosis respuesta, acorde a la prueba de correlación de Pearson, no se observó actividad en relación con la dosis $(p>0.05)$.

Tabla 3. Tiempos de reacción en la prueba de inmersión de la cola según la dosis

\begin{tabular}{|c|c|c|c|c|c|c|c|}
\hline $\begin{array}{l}\text { Tiempo } \\
\text { (minuto) }\end{array}$ & $\begin{array}{c}\text { Metformina } \\
50 \mathrm{mg} / \mathrm{kg}^{*} \\
\text { TR }\end{array}$ & $\begin{array}{l}\text { Metformina } \\
100 \mathrm{mg} / \mathrm{kg}^{*}\end{array}$ & $\begin{array}{l}\text { Metformina } \\
150 \mathrm{mg} / \mathrm{kg}^{*}\end{array}$ & $\begin{array}{l}\text { Metformina } \\
200 \mathrm{mg} / \mathrm{kg}^{*}\end{array}$ & $\begin{array}{l}\text { Metformina } \\
250 \mathrm{mg} / \mathrm{kg}^{*}\end{array}$ & $\begin{array}{c}\text { Diclofenaco } \\
8 \mathrm{mg} / \mathrm{kg}^{*} \\
\text { TR }\end{array}$ & Blanco \\
\hline Pre & 3.35 & 3.42 & 4.57 & 3.78 & 3.81 & 3.02 & 5.56 \\
\hline 0 & 3.88 & 5.25 & $8.28^{* *}$ & 4.45 & 5.64 & $8.11^{* * *}$ & 4.47 \\
\hline 15 & 4.45 & $7.39^{* *}$ & $13.73^{\star *}$ & 6.15 & 6.67 & $14.81^{* \star *}$ & 4.69 \\
\hline 30 & 3.65 & 7.61 & $12.19^{\star *}$ & 6.63 & $8.28^{* *}$ & $14.91^{* * *}$ & 5.01 \\
\hline 45 & 4.69 & $10.92^{* *}$ & $13.49^{* *}$ & $7.48^{* *}$ & $9.58^{* *}$ & $15.00^{* * *}$ & 4.30 \\
\hline 60 & $7.19^{* *}$ & $12.60^{* *}$ & $14.55^{\star *}$ & $8.47^{* *}$ & $10.99^{* *}$ & $14.89^{* * *}$ & 4.41 \\
\hline 120 & 6.09 & $10.71^{* *}$ & $13.78^{* *}$ & $8.29^{* *}$ & $10.62^{* *}$ & $14.43^{* * *}$ & 4.46 \\
\hline
\end{tabular}

TR: Tiempo de reacción en segundos al estímulo físico nociceptivo. *Prueba de ANOVA de una cola $p<0.05 .{ }^{* *}$ Prueba de Tukey $p<0.05$. ***Prueba de Tukey $p>0.05$.

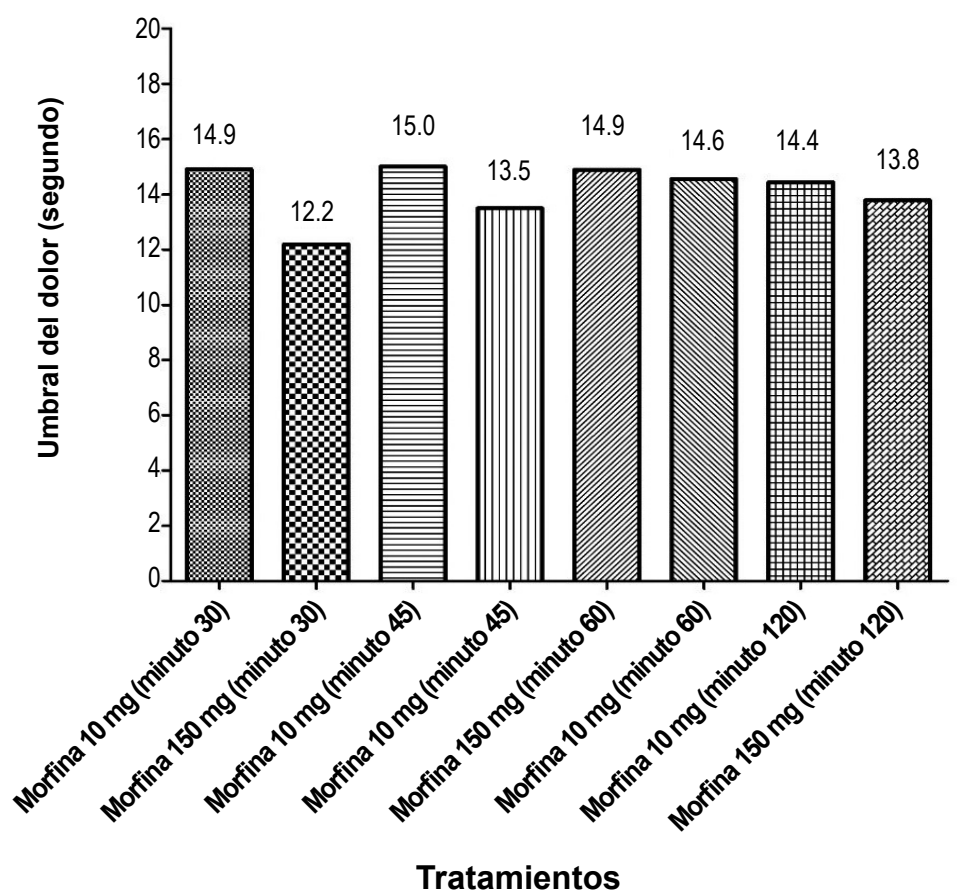

Figura 2. Promedios de tiempo de reacción y dosis en la prueba de la placa caliente 


\section{DISCUSIÓN}

La prueba de edema inducido por carragenina es ampliamente usada en modelos experimentales de inflamación aguda en ratones (21-23). La inflamación producida por carragenina sigue la vía de Bcl10, NF$\kappa \mathrm{B}$ y IkBa, que resulta en la liberación de mediadores inflamatorios en dos fases. Durante la primera fase (1 hora después de la inyección de carragenina) se liberan histamina, serotonina y citoquinas; y en la segunda, bradiquininas, proteasas, prostaglandinas y lisosomas. La última etapa es la más sensible frente a los agentes antiinflamatorios de uso clínico ${ }^{(24)}$, como el diclofenaco, que actúa inhibiendo la síntesis de prostaglandinas al inhibir la ciclooxigenasa 1 y $2{ }^{(25)}$.

La actividad antiinflamatoria de metformina solo fue significativa durante la segunda y tercera hora en algunas dosis, este efecto podría deberse a su actividad, in vitro, de evitar la fosforilación de $\mathrm{I} \mathrm{B}$, y la activación de las vías NF-KB ${ }^{(12,26,27)}$ y del Egr-1 ${ }^{(28)}$. En consecuencia, se inhibe la síntesis sVCAM, sICAM, vWF, IL-1, IL-6, IL-8 y TNFa ${ }^{(29-31)}$, acción que se confirma por la hora en que se presentan los efectos ${ }^{(24)}$.

Notamos que no se obtuvo una correlación dosis-efecto, y el mayor efecto se consiguió con la dosis más baja. Esto podría deberse a factores farmacocinéticos y farmacodinámicos como la expresión de receptores diana, la sensibilidad, saturación, metabolismo, intervención hormonal, entre otros ${ }^{(32)}$. Además, los resultados son comparables con otros estudios in vivo que siguieron la misma metodología ${ }^{(15,21,23)}$.

En la evaluación del efecto antinociceptivo de la metformina se utilizaron dos modelos experimentales de dolor: prueba de placa caliente y prueba de inmersión de la cola, que se caracterizan porque evalúan el efecto analgésico directamente en la vía del dolor, y sin presencia de mediadores de la inflamación o injuria ${ }^{(14)}$.

Las pruebas utilizadas estimulan una respuesta a nivel supraespinal, causada por activación de los receptores nerviosos térmicos de la piel, que responden ante los aumentos de temperatura $\mathrm{y}$, finalmente, es percibido como dolor ${ }^{(33)}$. En las ratas, las manifestaciones clínicas en la prueba de placa caliente son lamerse las patas (que solo se inhibe con el uso de agonistas opioides) y saltar (que se inhibe por analgésicos y antiinflamatorios no esteroides) (34). Mientras que en la prueba de inmersión de cola se evidencia el reflejo de retirada de cola, que es sensible a los compuestos opioides como la morfina ${ }^{(14)}$.

El efecto antinociceptivo de metformina en la prueba de placa caliente fue principalmente significativo en dosis de
$200 \mathrm{mg} / \mathrm{kg}$ y $250 \mathrm{mg} / \mathrm{kg}$ a la segunda hora, incluso, estas dosis tuvieron mayor efecto en el TR a comparación al diclofenaco. El resultado de la prueba de inmersión de cola fue significativo a partir de la dosis de $100 \mathrm{mg} / \mathrm{kg} \mathrm{y}$, al compararse el efecto de metformina $150 \mathrm{mg} / \mathrm{kg}$ con el mejor resultado obtenido por morfina, se encontró efecto antinociceptivo similar. El mecanismo de acción probable de la metformina en el dolor agudo puede estar relacionado al bloqueo de la generación de impulsos de nociceptores periféricos, bloqueo de la transmisión sináptica de los impulsos de dolor en el sistema nervioso central (14), o a un mecanismo central aún desconocido ${ }^{(35)}$. En contraste a los resultados obtenidos, otros estudios no atribuyen actividad analgésica significativa a la metformina ${ }^{(36,37)}$.

Hay pocos estudios experimentales in vivo que evalúen el efecto antinociceptivo o antiinflamatorio de la metformina $(15,30)$. Sugerimos continuar las investigaciones sobre sus efectos pleiotrópicos, ya que este fármaco es ampliamente usado por diabéticos que, a su vez, es un grupo numeroso y vulnerable a desarrollar enfermedades inflamatorias (38) y dolor neuropático ${ }^{(39)}$, entre otros.

Una primera conclusión, es que se demuestra el efecto antiinflamatorio en el componente de edema y la actividad antinociceptíva de la metformina a diferentes dosis, en modelos experimentales de inflamación y dolor agudo en ratones.

Ante la evidencia presentada por el presente estudio y sus precedentes, es necesario continuar la investigación respecto de los efectos sobre el cuadro agudo de dolor e inflamación de metformina, ya que podrían significar una alternativa para su control efectivo.

El control del dolor y la inflamación en los cuadros agudos con fármacos alternativos a los AINES u opioides, con un medicamento como la metformina es importante, pues los efectos secundarios y adversos conocidos por los fármacos vigentes, hacen relevante la búsqueda de nuevas alternativas.

Si bien los pacientes con enfermedades cardiometabólicas son susceptibles de padecer enfermedades inflamatorias, existen múltiples patologías crónicas que los afectan con exacerbaciones agudas de dolor e inflamación, como en los principales cuadros reumatológicos como artritis reumatoide, fibromialgia, entre otros. Estos pacientes podrían beneficiarse con fármacos derivados de investigaciones de nuevas drogas, o usos nuevos de fármacos ya existentes.

La metformina ha demostrado ser un normoglicemiante oral efectivo y seguro para los pacientes durante más de 60 años. La investigación sobre sus beneficios en la 
reducción de factores inflamatorios, en la que presente estudio contribuye, avizora una prometedora expansión.

\section{REFERENCIAS BIBLIOGRÁFICAS}

1. Paul SM, Mytelka DS, Dunwiddie CT, Persinger CC, Munos $\mathrm{BH}$, Lindborg SR, et al. How to improve R\&D productivity: the pharmaceutical industry's grand challenge. Nat Rev Drug Discov. 2010; 9(3): 203-14.

2. Munos B. Lessons from 60 years of pharmaceutical innovation. Nat Rev Drug Discov. 2009; 8(12): 959-68.

3. Keiser MJ, Setola V, Irwin JJ, Laggner C, Abbas A, Hufeisen SJ, et al. Predicting new molecular targets for known drugs. Nature. 2009; 462(7270): 175-81.

4. Wolff T, Miller T, Ko S. Aspirin for the primary prevention of cardiovascular events: an update of the evidence for the U.S. preventive services task force. Ann Intern Med. 2009; 150(6): 405-10.

5. Sirven Jl. New uses for older drugs: the tales of aspirin, thalidomide, and gabapentin. Mayo Clin Proc. 2010; 85(6): 508-11.

6. Cuzick J, Otto F, Baron JA, Brown PH, Burn J, Greenwald P, et al. Aspirin and non-steroidal anti-inflammatory drugs for cancer prevention: an international consensus statement. Lancet Oncol. 2009; 10(5): 501-7.

7. Mancera-Romero J, Hormigo-Pozo A, Fernández-Arquero J, Baca-Osorio A, Aparicio-Cervantes MJ, Muñoz-González L. Use of glucose-lowering drugs in a primary care setting in Malaga during the years 2008-2012. Semergen. 2014; 40(1): 4-11.

8. Scarpello $\mathrm{JH}$, Howlett HC. Metformin therapy and clinical uses. Diab Vasc Dis Res. 2008; 5(3): 157-67.

9. Lamanna C, Monami M, Marchionni N, Mannucci E. Effect of metformin on cardiovascular events and mortality: a metaanalysis of randomized clinical trials. Diabetes Obes Metab. 2011; 13(3): 221-8.

10. Hoeger K, Davidson K, Kochman L, Cherry T, Kopin L, Guzick DS. The Impact of metformin, oral contraceptives, and lifestyle modification on polycystic ovary syndrome in obese adolescent women in two randomized, placebo-controlled clinical trials. J Clin Endocrinol Metab. 2008; 93(11): 4299306.

11. Alexánderson-Rosas E, Martínez AJ, Ochoa-López JM, CallejaTorres R, Sierra-Fernández C, Iñarra-Talboy F, et al. Efecto del tratamiento combinado con metformina/glimepirida sobre la función endotelial en pacientes con diabetes mellitus tipo 2 evaluados mediante PET. Arch Cardiol México. 2009; 79(4): 149-256.

12. Caballero AE, Delgado A, Aguilar-Salinas CA, Herrera AN, Castillo JL, Cabrera T, et al. The differential effects of metformin on markers of endothelial activation and inflammation in subjects with impaired glucose tolerance: a placebo-controlled, randomized clinical trial. J Clin Endocrinol Metab. 2004; 89(8): 3943-8.

13. Guppy A, Jamal-Hanjani M, Pickering L. Anticancer effects of metformin and its potential use as a therapeutic agent for breast cancer. Future Oncol. 2011; 7(6): 727-36.

14. Wiley J. Current Protocols in Pharmacology [Internet]. Disponible en: http://onlinelibrary.wiley.com/ book/10.1002/0471141755/homepage/Archive.html
15. Montes J, Pacheco K, Figueroa J, Inga V, Ortega Y, Flores C, et al. Análisis de la actividad antiinflamatoria y analgésica aguda de la metformina mediante el Test de Formalina. Horiz Med. 2012; 12(2).

16. Galduf J, Gallego C, Escrivá J, Montserrat V. Ensayos clínicos: Elaboración de una lista-guía para la valoración de protocolos. Farm Hosp. 1995; 19(1): 17-23.

17. Mujica PY. Procesamiento de señales in vivo, edición audivisual y creación de un prototipo de software educativo para simulación de experimentos en farmacología [Tesis]. Universidad San Martín de Porres: Lima. Facultad de Medicina Humana; 2009.

18. Van Wilgenburg H. Microlabs for Pharmacologists [Internet]. Disponible en: https://norecopa.no/norina/microlabs-forpharmacologists

19. National Research Council (US) Institute for Laboratory Animal Research. International Guiding Principles for Biomedical Research Involving Animals (1985: Washington (DC): National Academies Press US; 2004.

20. Zimmermann M. Ethical guidelines for investigations of experimental pain in conscious animals. Pain. 1983; 16(2): 109-10.

21. Chi SC, Jun HW. Anti-inflammatory activity of ketoprofen gel on carrageenan-induced paw edema in rats. J Pharm Sci. 1990; 79(11): 974-7.

22. Matsumoto K, Obara S, Kuroda Y, Kizu J. Anti-inflammatory effects of linezolid on carrageenan-induced paw edema in rats. J Infect Chemother. 2015; 21(12): 889-91.

23. Li YY, Huang SS, Lee MM, Deng JS, Huang GJ. Anti-inflammatory activities of cardamonin from Alpinia katsumadai through heme oxygenase-1 induction and inhibition of NF-KB and MAPK signaling pathway in the carrageenan-induced paw edema. Int Immunopharmacol. 2015; 25(2): 332-9.

24. Necas J, Bartosikova L. Carrageenan: a review. Vet Med (Praha). 2013; 58(4): 187-205.

25. Gan TJ. Diclofenac: an update on its mechanism of action and safety profile. Curr Med Res Opin. 2010; 26(7): 1715-31.

26. Hattori Y, Suzuki K, Hattori S, Kasai K. Metformin inhibits cytokine-induced nuclear factor $\mathrm{kb}$ activation via AMPactivated protein kinase activation in vascular endothelial cells. Hypertension. 2006; 47(6): 1183-8.

27. Koh SJ, Kim JM, Kim IK, Ko SH, Kim JS. Anti-inflammatory mechanism of metformin and its effects in intestinal inflammation and colitis-associated colon cancer. J Gastroenterol Hepatol. 2014; 29(3): 502-10.

28. Arai M, Uchiba M, Komura H, Mizuochi Y, Harada N, Okajima K. Metformin, an antidiabetic agent, suppresses the production of tumor necrosis factor and tissue factor by inhibiting early growth response factor-1 expression in human monocytes in vitro. J Pharmacol Exp Ther. 2010; 334(1): 206-13.

29. Isoda K, Young JL, Zirlik A, MacFarlane LA, Tsuboi N, Gerdes $\mathrm{N}$, et al. Metformin inhibits proinflammatory responses and nuclear factor-kb in human vascular wall cells. Arterioscler Thromb Vasc Biol. 2006; 26(3): 611-7.

30. Gómez-García A, Martínez Torres G, Ortega-Pierres LE, Rodríguez-Ayala E, Álvarez-Aguilar C. Rosuvastatina y metformina reducen la inflamación y el estrés oxidativo en pacientes con hipertensión y dislipemia. Rev Esp Cardiol. 2007; 60(12): 1242-9.

31. Deans KA, Sattar N. "Anti-inflammatory" drugs and their 
effects on type 2 diabetes. Diabetes Technol Ther. 2006; 8(1): 18-27.

32. Brunton LL, Lazo JS, Parket KL. Goodman \& Gilman: Las bases farmacológicas de la terapéutica. 12da ed. McGraw Hill Mexico; 2012.

33. Hall JE. Guyton y Hall. Tratado de fisiología médica. 12da ed. Elsevier Health Sciences; 2011.

34. Le Bars D, Gozariu M, Cadden SW. Animal models of nociception. Pharmacol Rev. 2001; 53(4): 597-652.

35. Verma S, Mundkinajeddu D, Agarwal A, Chatterjee SS, Kumar V. Effects of turmeric curcuminoids and metformin against central sensitivity to pain in mice. J Tradit Complement Med. 2016; 7(2): 145-51.

36. Ortiz MI. Blockade of the antinociception induced by diclofenac, but not of indomethacin, by sulfonylureas and biguanides. Pharmacol Biochem Behav. 2011; 99(1): 1-6.

37. Ortiz MI. Metformin and phenformin block the peripheral antinociception induced by diclofenac and indomethacin on the formalin test. Life Sci. 2012; 90(1-2): 8-12.

38. Ananthakrishnan AN, Cagan A, Cai T, Gainer VS, Shaw SY, Churchill $S$, et al. Diabetes and the risk of infections with immunomodulator therapy in inflammatory bowel diseases. Aliment Pharmacol Ther. 2015; 41(11): 1141-8.

39. Dermanovic DV, Hrabac P, Skegro D, Smiljanic R, Dobrota S, Prkacin I, et al. The impact of neuropathic pain and other comorbidities on the quality of life in patients with diabetes. Health Qual Life Outcomes. 2014; 12(1): 171.

Fuentes de financiamiento:

Este artículo ha sido financiado por los autores.

\section{Conflicto de interés:}

Los autores declaran no tener ningún conflicto de interés.

\section{Correspondencia:}

Alberto Alcibíades Salazar Granara

Dirección: Av. El Corregidor $N^{\circ} 1531$, Las Viñas, La Molina. Lima, Perú. Teléfono: 511 3652300, anexo 151.

Correo electrónico: asalazarg@usmp.pe

\section{Recibido: 25 de mayo de 2019 \\ Evaluado: 08 de julio de 2019 \\ Aprobado: 18 de julio de 2019.}

๔ La revista. Publicado por Universidad de San Martín de Porres, Perú. (c) Br Licencia de Creative Commons Artículo en acceso abierto bajo términos de Licencia Creative Commons Atribución 4.0 Internacional. (http://creativecommons.org/licenses/by/4.0/)

\section{ORCID iDs}

Joseph Sánchez Gavidia

Elmer Lujan Carpio

Carlos Pante Medina

Alberto Salazar Granara https://orcid.org/0000-0001-8724-3694 https://orcid.org/0000-0003-3034-5061 https://orcid.org/0000-0002-8896-4298 https://orcid.org/0000-0003-1996-3176 